\title{
28 Research Square \\ Identification of A New Biomarker For Herpes Zoster Infection in Rheumatoid Arthritis
}

Marowa Hashimoto ( $\square$ mhashimoto@leaf.ocn.ne.jp )

Research Institute of Joint Diseases

\section{Keiko Funahashi}

Matsubara Mayflower Hospital

\section{Ken Tsumiyama}

Matsubara Mayflower Hospital

Yoshinori Takashima

Kobe University Graduate School of Medicine

Toshihisa Maeda

Kobe University Graduate School of Medicine

Koji Fukuda

Matsubara Mayflower Hospital

Shinya Hayashi

Kobe University Graduate School of Medicine

Ryosuke Kuroda

Kobe University Graduate School of Medicine

Tsukasa Matsubara

Matsubara Mayflower Hospital

\section{Research Article}

Keywords: biomarker, infection, rheumatoid arthritis, Herpes zoster

Posted Date: June 25th, 2021

DOI: https://doi.org/10.21203/rs.3.rs-651045/v1

License: (c) (i) This work is licensed under a Creative Commons Attribution 4.0 International License.

Read Full License 


\section{Abstract}

Herpes zoster $(\mathrm{HZ})$ is known as a side effect of using biologics in rheumatoid arthritis (RA). Incidence of this side effect may be different depending on genetic factors because susceptibility to HZ infection varies by race. Here, we analyzed the statistical relationships of whole genome single nucleotide polymorphisms (SNPs) with HZ infection in biologics-treated RA patients.

The subjects were 321 Japanese female patients (including 56 herpes virus infected patients) of RA using biologics. The relationships of 302,814 SNPs with $\mathrm{HZ}$ infection were analyzed using case-control

analyses by Fisher's exact tests. We picked up SNPs $\left(P<10^{-8}\right)$ significantly associated with $\mathrm{HZ}$ infection. Then, herpes infection was compared among the genotypes using a multivariate logistic regression analysis adjusted for onset age of RA.

Rs10774580 located in 2'-5'-oligoadenylate synthetase like gene (OASL) was significantly associated with herpes virus infection. The minor allele homozygous carrier was positively associated with herpes virus infection in multivariate analysis.

We for the first time showed a significant relationship between a genetic factor and $\mathrm{HZ}$ infection among RA patients. Rs 10774580 may be one of the biomarkers for $\mathrm{HZ}$ infection.

\section{Introduction}

Rheumatoid arthritis (RA) is a progressive autoimmune disease well defined by widely accepted symptoms such as chronic joint inflammation and structural damage ${ }^{1}$. In treatment for RA at present, using biological agents such as tumor necrosis factor (TNF), interleukin-6 (IL-6) and cytotoxic Tlymphocyte-associated protein 4 (CTLA-4) blockades is extremely useful because these agents specifically inhibit immune responses and inflammation. On the other hand, because these agents are immunosuppressant, infectious diseases become a significant problem in treatment for RA.

Herpes zoster $(\mathrm{HZ})$ is one of the most common viral infections in treatment for RA with immunosuppressants such as biological agents. In fact, it has been reported that RA patients have a higher risk of $\mathrm{HZ}$ compared with the general population $2,3,4$. Therefore, a number of studies analyzed relationships of the incidence of $\mathrm{HZ}$ with various possible risk factors. As the result, aging, high disease activity and corticosteroid, methotrexate and biological agents were reported as the risk for $\mathrm{HZ}^{5}$. These studies, however, have not taken genetic factors into consideration. On the other hand, several studies have identified genetic loci associated with onset of RA ${ }^{6}$. It has also been reported that the effectiveness of biologic agents can be predicted by combination of single nucleotide polymorphisms (SNPs) ${ }^{7}$. These studies used genome-wide association study (GWAS) in order to identify the genetic factors. Thus, conducting GWAS is thought to be valuable in order to identify unknown genetic factors associated with $\mathrm{HZ}$ in RA. 
In this study, in order to identify SNPs associated with $\mathrm{HZ}$ infection, we analyzed the statistical relationships of whole genome SNPs with $\mathrm{HZ}$ infection among biological agents-treated RA patients.

\section{Results}

The basic characteristics of the patients are presented in Table 1 . The total patients were 320 aged 45.5 \pm 13.9 years (mean \pm SD).

Only one SNP was identified that was significantly associated with $\mathrm{HZ}$ infection (Fig. 1). The SNP was rs10774580 in 2'-5'-oligoadenylate synthetase like gene (OASL).

Table 2 presents the relationships of OASL genotype and onset age of RA with HZ infection. The minor allele homozygous of rs10774580 and the onset age of RA ( $\geq 65$ years) were positively associated with $\mathrm{HZ}$ infection. Adjusted OR for the minor allele homozygous was 15.6 (95 \% Cl $3.9-61.4$ ). Adjusted OR for the onset age ( $\geq 65$ years) was $2.6(95 \% \mathrm{Cl} 1.1-6.4)$.

\section{Discussion}

To our knowledge, our study is the first to analyze the relationships of whole genome SNPs with $\mathrm{HZ}$ infection among bDMARDS-treated RA patients and to identify a SNP as one of the biomarkers for HZ infection.

It has been reported that aging, high disease activity, corticosteroid use and the use of methotrexate are risk factors for $\mathrm{HZ}$ infection in RA patients ${ }^{5}$. In addition, it has been reported that susceptibility to $\mathrm{HZ}$ infection in RA varies by race ${ }^{8}$. In fact, Japanese and Taiwanese have a higher risk compared to Americans and Europeans ${ }^{9}$. In this regard, genetic background may also affect the susceptibility. However, because there are not studies that take into account genetic polymorphisms such as SNPs, it is likely that genetic polymorphisms associated with the susceptibility were overlooked. Therefore, we conducted GWAS which is powerful tool to collectively identify SNPs associated with the susceptibility.

In our study, rs10774580 located in intron region of OASL gene was significantly associated with $\mathrm{HZ}$ infection. The minor allele homozygous were positively associated with $\mathrm{HZ}$ infection. Human OASL has an antiviral activity against RNA viruses ${ }^{10,11}$. On the other hand, OASL inhibits type I interferon (IFN) induction during DNA virus infection such as herpes simplex, vaccinia and adenovirus ${ }^{12}$. This is because OASL binds to cylic GMP-AMP synthase (cGAS) known as DNA sensor, and inhibits cyclic GMP-AMP (cGAMP) synthesis in cGAS-STING (stimulator of interferon gene) pathway sensing the majority of DNA viruses $^{12}$. Inhibiting IFN induction leads to enhancing DNA virus replication. Therefore, rs10774580 may affect the transcription of OASL because this SNP is intronic variation without amino acid substitution. As the result, the expression levels among OASL genotypes vary, and then differences of the susceptibility may be caused. 
Interestingly, several previous studies revealed that using Janus Kinase (JAK) inhibitors increased the risk of $\mathrm{HZ}$ infection compared to bDMARDS ${ }^{13,14}$. In this regard, it is unclear if rs 10774580 is also associated with $\mathrm{HZ}$ infection in JAK inhibitors-treated RA patients. Thus, further analyses are needed in JAK inhibitors-treated RA patients.

This study has several limitations. First, rs 10774580 was identified by the result of GWAS among Japanese RA patients. It is well known that allele frequencies of most SNPs vary in different ethnic groups. The allele frequency of rs 10774580 we identified also varied compared with the allele frequency of other ethnic groups reported in the HapMap database (https://www.ncbi.nlm.nih.gov/snp). Therefore, rs 10774580 may not be applicable to non-Japanese RA patients as the biomarker. A second limitation is that this study didn't take into consideration the incidence of $\mathrm{HZ}$ in each patient. It is well known that some RA patients repeatedly develop HZ. Therefore, in order to identify the other biomarkers, further studies taking in the incidence of $\mathrm{HZ}$ into consideration are desired.

\section{Patients And Methods}

Patients. We recruited 321 Japanese female patients of RA receiving treatment with biological diseasemodifying antirheumatic drugs (bDMARDs). They included $56 \mathrm{HZ}$ infected patients. Written informed consent to participate in this study was obtained from each patient. This study was approved by the ethical committee for analytical research on the human genome of the Matsubara Mayflower Hospital. All methods were performed in accordance with relevant guidelines and regulations.

Genome-wide SNP genotyping. The patients' whole blood samples were used for DNA extraction at Mitsubishi BCL Inc. Genome wide SNP genotyping were performed at deCode genetics Inc. (Reykjavic, Iceland) using Illumina HumanHap300K chip technology (Illumina Corp., San Diego, CA, USA). After genotyping, 302,814 of 317,503 SNPs excluded SNPs with call rates $<90 \%$ and minor allele frequency $<1$ $\%$ were used in the case-control analysis described below.

Statistical analysis. We used case-control analysis to analyze the relationship of 302,814 SNPs with onset of HZ by Fisher's exact tests using SVS 8.1.1 (Golden Helix Inc.). After case-control analyses, we picked up SNPs significantly associated with $\mathrm{HZ}$ infection. Univariate and multivariate logistic regression analyses were used to examine the effects of the SNP and onset age of RA on the risk for $\mathrm{HZ}$ infection. The logistic regression analyses were carried out using EZR ${ }^{15}$ (Saitama Medical Center, Jichi Medical University, Saitama, Japan). EZR is a graphical user interface for R (The R Foundation for Statistical Computing, Vienna, Austria, version 2.13.0). $P$ values $<10^{-8}$ were considered significant in case-control analysis. $P$ values $<0.05$ were also considered significant in the logistic regression analyses.

\section{Conclusion}

This is the first report of a significant association between a genetic factor and $\mathrm{HZ}$ infection among bDMARDS-treated RA patients. As the result of GWAS, we showed that rs 10774580 in OASL gene was 
significantly associated with $\mathrm{HZ}$ infection. Therefore, this SNP may be one of the biomarkers for predicting $\mathrm{HZ}$ infection among RA patients before using biologics.

\section{Declarations}

\section{Acknowledgements}

We would like to thank Dr. Eisuke Shono, Tomomaro Izumihara, Tomomi Tsuru and Motohiro Oribe for offering the patients' whole blood samples. We also would like to thank Ms. Carol Matsubara for proofreading the manuscript.

\section{Author contributions}

M.H., K.Fun., K.T., Y.T., T.Mae., K.Fuk., S.H., R.K. and T.Mat. participated in the study design. K.Fun. contributed to data collection. M.H. carried out the statistical analyses. M.H. and T.Mat contributed to drafting of the manuscript. M.H. and T.Mat. were responsible for the analysis and interpretation of data.

\section{Competing Interests}

The authors declare that they have no competing interests.

\section{References}

1. Combe, B. Early rheumatoid arthritis: strategies for prevention and management. Best Practice and Research: Clinical Rheumatology 21, 27-42 (2007).

2. Antonelli, M. A. S., Moreland, L. W. \& Brick, J. E. Herpes zoster in patients with rheumatoid arthritis treated with weekly, low-dose methotrexate. The American Journal of Medicine 90, 295-298 (1991).

3. Smitten, A. L. et al. The risk of herpes zoster in patients with rheumatoid arthritis in the United States and the United Kingdom. Arthritis Care Res. 57, 1431-1438 (2007).

4. Veetil, B. M. A. et al. Incidence and time trends of herpes zoster in rheumatoid arthritis: A populationbased cohort study. Arthritis Care Res. 65, 854-861 (2013).

5. Nakajima, A. et al. Incidence of herpes zoster in japanese patients with rheumatoid arthritis from 2005 to 2010. Mod. Rheumatol. 25, 558-561 (2015).

6. Okada, Y. et al. Meta-analysis identifies nine new loci associated with rheumatoid arthritis in the Japanese population. Nat. Genet. 44, 511-516 (2012).

7. Hashimoto, M. et al. Algorithms using genome-wide association studies for prediction of effectiveness of biologics in rheumatoid arthritis. Pers. Med. Universe 9, 20-26 (2020).

8. Winthrop, K. L. et al. Herpes Zoster and Tofacitinib: Clinical Outcomes and the Risk of Concomitant Therapy. ARTHRITIS Rheumatol. 69, 1960-1968 (2017).

9. Kawai, K., Gebremeskel, B. G. \& Acosta, C. J. Systematic review of incidence and complications of herpes zoster: towards a global perspective. BMJ Open 4, 4833 (2014). 
10. Zhu, J. et al. Antiviral activity of human oligoadenylate synthetases-like (OASL) is mediated by enhancing retinoic acid-inducible gene I (RIG-I) signaling. Immunity 40, 936-948 (2014).

11. Dhar, J. et al. 2'-5'-Oligoadenylate Synthetase-Like Protein Inhibits Respiratory Syncytial Virus Replication and Is Targeted by the Viral Nonstructural Protein 1. J. Virol. 89, 10115-10119 (2015).

12. Ghosh, A. et al. Oligoadenylate synthetases family protein OASL inhibits DNA sensor cGAS activity during DNA virus infection to limit interferon production. HHS Public Access. Immunity 50, 51-63 (2019).

13. Cohen Jeffrey Curtis Ryan DeMasi Yan Chen Haiyun Fan Arif Soonasra Roy Fleischmann, S. R. Worldwide, 3-Year, Post-Marketing Surveillance Experience with Tofacitinib in Rheumatoid Arthritis. Rheumatol. Ther. 5,

14. Bechman, K. et al. A systematic review and meta-analysis of infection risk with small molecule JAK inhibitors in rheumatoid arthritis. Rheumatol. (United Kingdom) 58, 1755-1766 (2019).

15. Kanda, Y. Investigation of the freely available easy-to-use software \&lsquo;EZR\&rsquo; for medical statistics. Bone Marrow Transplant. (2013). doi:10.1038/bmt.2012.244

\section{Tables}

Table 1. Characteristics of patients.

\begin{tabular}{|ll|}
\hline \multicolumn{2}{|c|}{ variables } \\
\hline Number of patients (\%) & 321 \\
\hline Non herpes zoster & $265(82.6)$ \\
\hline Herpes zoster & $56(17.4)$ \\
\hline Onset age (years) & $45.5 \pm 13.9$ \\
\hline$\geq 65$ years & $26(8.1)$ \\
\hline$<65$ years & $257(80.1)$ \\
\hline unknown & $38(11.8)$ \\
\hline OASL genotype (\%) & $186(58.0)$ \\
\hline major allele homozygous & $122(38.0)$ \\
\hline heterozygous & $13(4.0)$ \\
\hline minor allele homozygous & \\
\hline Values are mean \pm SD, number of the patients \\
\hline
\end{tabular}


Table 2. Relationships between the OASL genotypes and onset age with $\mathrm{HZ}$ infection.

\begin{tabular}{|lllll|}
\hline \multicolumn{1}{|l}{ Variables (high-risk group) } & \multicolumn{2}{l}{ Univariate } & \multicolumn{2}{l|}{ Multivariate $^{\mathrm{a}}$} \\
\cline { 2 - 5 } & OR & $95 \% \mathrm{Cl}$ & OR & $95 \% \mathrm{Cl}$ \\
\hline OASL genotype (minor allele homozygous) & 19.0 & $5.0-71.6$ & 15.6 & $3.9-61.4$ \\
\hline Onset age ( $\geq 65$ years) & 2.1 & $0.8-5.3$ & 2.6 & $1.1-6.4$ \\
\hline Abbreviation: OR, odds ratio. ${ }^{a}$ Adjusted for all variables & & \\
\hline
\end{tabular}

\section{Figures}

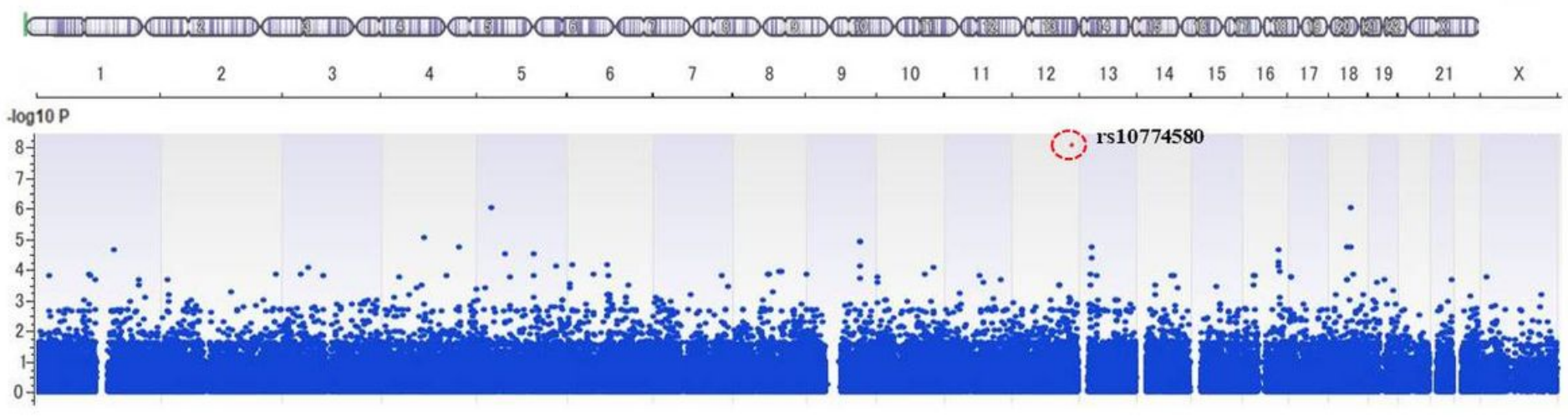

Figure 1

Manhattan plot showing Fisher's exact tests' results. 